\title{
Clinical and Microbiological Epidemiology of Otomycosis in the Centre Region of Cameroon
}

\author{
Ekpo Alfred Itor ${ }^{1}$, Michel Noubom ${ }^{2}$, Claude Nangwat ${ }^{1}$, Dougue Aude Ngueguim ${ }^{3}$, \\ Cyrille Levis Kountchou ${ }^{1}$, Ngouana Kammalac Thierry ${ }^{4}$, Dzoyem Jean Paul, *, \\ Tume Christopher Bonglavnyuy,"* \\ ${ }^{1}$ Department of Biochemistry, Faculty of Science, University of Dschang, Dschang, Cameroon \\ ${ }^{2}$ Department of Biological Sciences, Faculty of Medicine and Pharmaceutical Sciences, University of Dschang, Dschang, Cameroon \\ ${ }^{3}$ Department of Biochemistry, University of Yaoundé 1, Yaoundé, Cameroon \\ ${ }^{4}$ Biomedical Research Unit, Sion Laboratory, Yaounde, Cameroon
}

\section{Email address:}

alfredekpo@ymail.com (E. A. Itor), noubommichel@yahoo.fr (M. Noubom), nangwatclaude@yahoo.com (C. Nangwat), audedougue@yahoo.fr (D. $\Lambda$. Ngueguim), cyrillekountchou@yahoo.com (C. L. Kountchou), ngouanathi@yahoo.com (N. K. Thierry), jpdzoyem@yahoo.fr (D. J. Paul), tumechrist@yahoo.com (T. C. Bonglavnyuy)

${ }^{*}$ Corresponding author

\section{To cite this article:}

Ekpo Alfred Itor, Michel Noubom, Claude Nangwat, Dougue Aude Ngueguim, Cyrille Levis Kountchou, Ngouana Kammalac Thierry, Dzoyem Jean Paul, Tume Christopher Bonglavnyuy. Clinical and Microbiological Epidemiology of Otomycosis in the Centre Region of Cameroon. European Journal of Clinical and Biomedical Sciences. Vol. 6, No. 5, 2020, pp. 78-83. doi: 10.11648/j.ejcbs.20200605.12

Received: August 13, 2020; Accepted: August 24, 2020; Published: September 7, 2020

\begin{abstract}
Otomycosis is a superficial, sub-acute or chronic infection of the external auditory canal, characterized by pruritis, inflammation, pain and itching commonly seen in tropical and subtropical regions of the world. Various host and environmental factors can predispose a person to otomycosis. However, a clinical presentation along with otoscopic observations of the patients shows fungal and bacterial infections. Proper identification of causative agents is necessary in order to prevent recurrences and complications such as hearing lost. The aim of our study was to determine the fungi and bacteria pathogens causing otomycosis and to derive association of risk factors with otomycosis of the clinically diagnosed patients. A descriptive cross-sectional study was conducted in the otorhinolaryngology department at the University Teaching hospital and the Central hospital over a period of one year. A total of 250 clinically diagnosed patients of otomycosis of age above one year were included in the study. We evaluated age and sex distribution, predisposing factors and complaints of the clinically diagnosed patients for otomycosis. All samples collected were transported and evaluated by both direct microscopic examination and culture method for bacteria and fungi examination, which were identified by standard procedures. Among 250 samples, $46.22 \%$ yielded fungal growth, $21.33 \%$ grew bacteria only and 32.44\% showed mixed growth of fungi and bacteria. Major fungal isolates were Aspergillus ( $\mathrm{n}=121)$ including 75 isolates of Aspergillus section Nigri, 20 isolates of Aspergillus section Flavi, 13 isolates of Aspergillus section Fumigati, 8 isolates of Aspergillus section Nudilante and 5 isolates of Aspergillus section Terrei. 48 isolates were identified as Candida species. Major bacterial isolates were Staphylococcus aureus $(\mathrm{n}=45)$ followed by Pseudomonas species $(\mathrm{n}=26)$, Klebsiella species $(\mathrm{n}=21)$, Escherichia coli $(\mathrm{n}=7)$ and Proteus species $(\mathrm{n}=3)$. This study highlights the highest isolation of Aspergillus section Nigri in cases of clinically diagnosed otomycosis patients at the two reference hospital in Yaoundé, Cameroon with high prevalence seen in patients using antibiotic eardrops as a mean of treatment from pains and itching.
\end{abstract}

Keywords: Otomycosis, Aspergillus Species, Candida Species, Bacterial Pathogens, Predisposing Factor

\section{Introduction}

Otomycosis is a sub acute or chronic fungal infection of the external auditory canal with some complications involving the middle ear [1]. It occurs because the protective lipid/acid balance of the ear is lost [2]. Otomycosis has a 
worldwide distribution. It is estimated that approximately $25 \%$ of the total cases of ear infections are due to fungi, and the disease is more prevalent in warm and humid climates [3]. In the recent years there has been an increased in the incidence as a result of possibly huge number of immunocompromised patients [4]. The fungus may not be the primary cause but merely a secondary invader in cases of otitis externa. Therefore otomycosis can be seen in mixed fungal with bacterial infections [5].

Bacterial infections are the most common cause of otitis externa in subtropical areas, followed by fungal infections, which are responsible for 10-20 percent of otitis externa cases [6]. Although a wide spectrum of fungi is involved, Aspergillus spp and Candida spp are the most common species encountered [7, 8]. Dematiaceous fungi such as Alternaria, Exophiala, Cladosporium and Nigrospora have also been described as causative agents of otomycosis [8-10]. Fungi are abundant in soil or sand that contains decomposing vegetable matter. These materials are desiccated rapidly in tropical sun and blown in the wind as small dust particles. The airborne fungal spores are carried by water vapour, a fact that correlates the higher rates of infection with the monsoon, during which the relative humidity rises to $80 \%$ [11].

Factors that predisposed to otitis externa include absence of cerumen, high humidity, increased temperature, bacterial otitis externa, corticoid therapy, swimming [12] and local trauma caused by sharp objects like sticks or hearing aids. Cerumen has a $\mathrm{pH}$ of 4 to 5 and so suppresses bacteria but conducive for fungal growth. Aquatic sports, including swimming and surfing, are particularly associated with otomycosis because repeated exposure to water results in removal of cerumen and drying of the external auditory canal [13]. Otomycosis is predominantly unilateral [14], found in all age groups, but majority of the cases of otomycosis occur in patients aged 2130 years with equal male - female distribution [15].

Yaoundé is a large town in the Centre Region of Cameroon. It is situated at the Southern plateau, the climate is equatorial consisting of 2 rainy season and 2 dry season with high temperature and high humidity throughout the year, a condition conducive for fungal and bacterial growth. Majority of patients attending the Central Hospital and University Teaching Hospital are agricultural labourers, in which their system of farming are bush fallowing and market gardening.

No epidemiological study has been done to identify the causative agents causing otomycosis in this geographical area. The present study has been undertaken to determine causative agents as well as the microbial distribution of pathogens involved in otomycosis in patients attending the ENT unit of Central Hospital and University Teaching Hospital with otitis externa.

\section{Materials and Methods}

\subsection{Study Type}

We carried out a descriptive cross sectional study on 250 patients clinically diagnosed to have otomycosis infection.
This was achieved by visiting the outpatient clinic of the otorhinolaryngology department weekly during a period of 12 months from March 2018 to February 2019 at the Central Hospital and University Teaching Hospital in Yaoundé, Cameroon. General information like age, sex, occupation, diabetic status, trauma, history of ear surgery or any fungal infection in other parts of body and laterality of symptoms were recorded. Any history of habits like use of oils/ear drops; wooden sticks or metal wax picks for removal of wax were also recorded.

\subsection{Ethical Consideration}

An Ethical clearance was obtained from the Centre Regional Committee for Human Health Research bearing no: 00842/CRERSHC/2018 and an authorization from the Directors of Central Hospital and University Teaching Hospital. Verbal informed consent was sought from individual patients from whom the ear samples were collected. To do this, it was explained to the patients in languages they understood that, the isolates that would be obtained from their samples would be used in this study. Samples from patients who consented were processed for bacteria and fungi growth at the Zion laboratory of Microbiology, Yaoundé. The ear samples from patients who disagreed were not included in the study.

\subsubsection{Inclusion Criteria}

All patients clinically diagnosed of otomycosis of age above one year presenting symptoms like itching, pain, feelings of blocked ear, tinnitus, deafness, discharge and in which otoscopic examinations reveals wet or dry masses of hyphae/spores were included in the study.

\subsubsection{Exclusion Criteria}

All patients presenting with symptoms of severe otitis media, tympanic membrane perforations, prior ear surgery were excluded in our study.

\subsubsection{Collection of Samples and Processing}

The samples were collected from the external auditory canal from patients that were clinically diagnosed to have otomycosis (figure 1) under aseptic conditions with the help of sterile cotton swab containing a preservation medium (Sigma transwab-liquid amies).

Samples were collected from either the right or left ear presenting signs or symptoms of otomycosis infection. All samples were evaluated by both direct examination and culture method. A portion of the sample was cultured on MacConkey agar, Chapman agar and Chocolates Agar plates at $37^{\circ} \mathrm{C}$ for $24 \mathrm{hrs}$ to $48 \mathrm{hrs}$ for bacterial growth. Identification of the bacterial isolates was done by standard bacteriological procedures [16]. For mycological identification, direct microscopic examination was carried out by $10 \% \mathrm{KOH}$ examination and inoculation of material was done on two prepared Sabouraud Dextrose Agar (SDA) plates supplemented with chloramphenicol. One plate was incubated at room temperature $25^{\circ} \mathrm{C}$ for $3-5$ days and another at $37^{\circ} \mathrm{C}$ for 48 hours. Both plates were observed for fungal 
growth daily. Fungal growth was identified by standard procedures [17]. Identification was done on the basis of colony morphology and lactophenol Cotton Blue (LPCB) mount microscopy. Aspergillus isolates were characterized by varying length of conidiophores and extent of coverage of vesicles by phialides. For characterization of Candida isolates, germ tube test was done by observing the production of germ tubes on isolates in serum after 2 hours of incubation at $37^{\circ} \mathrm{C}$.

\section{Results}

\subsection{Age-wise Distribution, Contributing Factors and Clinical Features of Otomycosis}

Out of 250 cases, 121 cases were in 21-40 years of age group constituting maximum of $(48.4 \%)$ of the cases clinically diagnosed of otomycosis (figure 2). We had 120 (48\%) males and $130(52 \%)$ females, giving a female predominance in otitis cases $(52 \%)$.

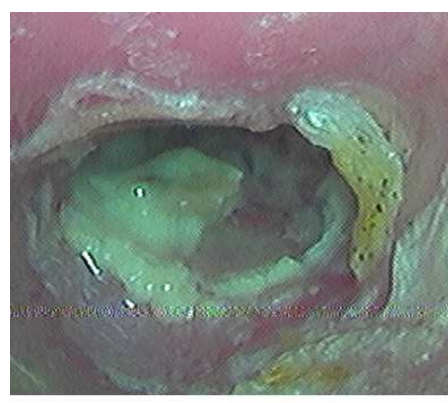

(a)

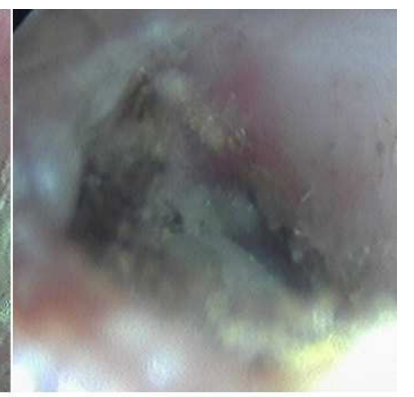

(b)
Figure 1. Otoscopic examination of fungi and bacteria isolates; (a) otoscopy of bacterial otitis (b) Otoscopy of Aspergillus niger mixed with Candida species otitis.

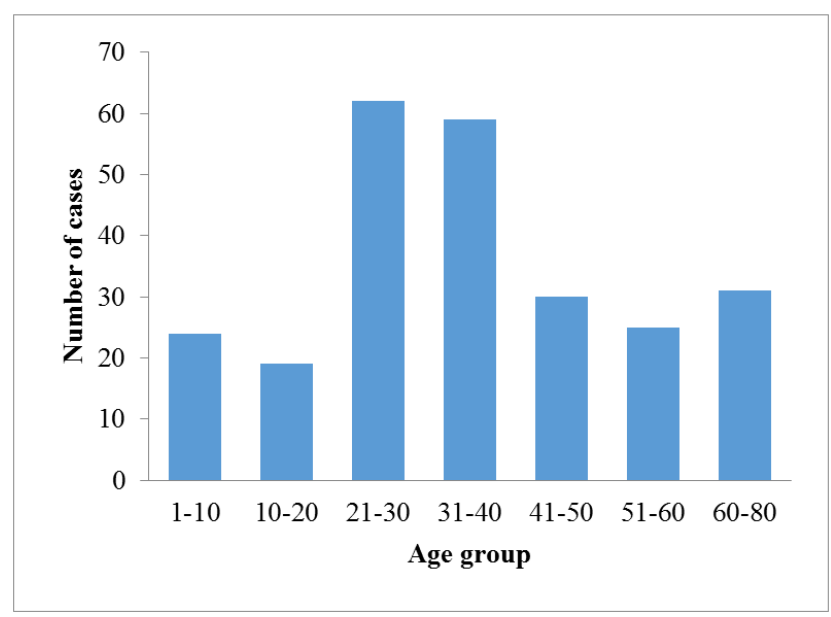

Figure 2. Age-wise distribution of the otomycosis cases.

Out of 250 patients 75 (30\%) had right ear involvement and $175(70 \%)$ had left ear involvement. In our study, 50 $(22.2 \%)$ cases had bilateral infection and 175 (77.8\%) cases had unilateral infection. There was a significant difference in right or left ear involvement. Majority of the patients gave the practice of using of antibiotic ear drops as means of treatment (Table 1). Most common presenting complaint was itching of the ear, followed by hearing difficulty, otalgia, ear discharge, inflammation and tinnitus (Table 1).

Table 1. Symptomatology and predisposing factors of otomycosis.

\begin{tabular}{lll}
\hline Major symptoms & Number of cases & Percentage (\%) \\
\hline Itching & 100 & 40 \\
Earpains (otalgia) & 50 & 20 \\
Discharge (otorrhea) & 30 & 12 \\
Tinnitus (ring in the ear) & 17 & 6.8 \\
Inflammation & 20 & 8 \\
Hearing difficulty & 33 & 13.2 \\
Total & 250 & 100 \\
\hline & & \\
\hline Predisposing factors & Number of cases & Percentage (\%) \\
\hline Antibiotic ear drops & 60 & 24 \\
Diabetes & 40 & 16 \\
Use of wooden object for & 30 & 12 \\
cleaning the ear & 13 & 5.2 \\
Swimming & 27 & 10.8 \\
Earpiece & 55 & 22 \\
No cerumen & 25 & 10 \\
No predisposing factor & 250 & 100 \\
Total & & \\
\hline
\end{tabular}

\subsection{Microbiological Findings}

Among 250 samples, 110 (44\%) showed only fungal elements, $81(32.4 \%)$ showed both bacterial and fungal elements and $34(13.6 \%)$ showed only bacteria. A total of $170(68 \%)$ samples showed fungal elements and $80(32 \%)$ showed bacteria. Among the 250 patients, $25(10 \%)$ of the samples didn't show either fungal elements or bacteria on microscopy and culture. Among 170 samples showing fungal elements on $\mathrm{KOH}$ mount, 168 samples grew fungi in culture. Also among 80 samples showing bacteria on Gram stain, 78 samples grew bacteria in culture. There was very little difference in direct microscopy findings and culture results. Among 250 samples, 80 samples yielded single fungus and 24 samples yielded two fungi each, without any bacterial isolates. Forty-eight samples grew single bacteria and five samples yielded two bacterial isolates each, without showing any fungal growth. All other samples yielded mixed fungal and bacterial growth. A total of 179 fungi were isolated from the 250 samples and 102 bacterial isolates were grown from 250 samples. From the 250 samples, 225 cases yielded a positive growth for both fungi and bacteria, with 25 samples not showing any growth.

The various fungi isolated from study group are shown in (Table 2) the commonest isolate was Aspergillus section Nigri constituting $44.38 \%$ of fungal isolates. Candida species constituting $28.40 \%$ was the second commonest isolate. With 20 isolates of Aspergillus section Flavi, 13 isolates of Aspergillus section Fumigati, 8 isolates of Aspergillus section Nidulante and 5 isolate of Aspergillus section Terrei. Aspergillus spp. made up for $71.60 \%$ against $28.40 \%$ of 
Candida species of the fungal isolated. Aspergillus species is the commonest fungus found in the atmosphere and a known contaminant everywhere. Conidia of these fungi spread through the air everywhere and wherever it finds suitable site for its growth it grows. When there is a predisposing condition for fungal growth in the external auditory canal, it is the commonest fungus to colonize and grow there. The various bacteria isolated from the study group is depicted in (Table 2) The major bacterial isolate was Staphylococcus aureus (44.12\%), followed by Pseudomonas spp. (25.49\%) and Klebsiella spp. (20.59\%). Escherichia coli $(6.86 \%)$ and Proteus spp. (2.94\%), are the other isolates. A total of 102 bacteria were isolated from 121 samples.

Table 2. Distribution of aetiological agents of otomycosis.

\begin{tabular}{lll}
\hline Fungi species & Number of isolates & Percentage (\%) \\
\hline Aspergillus section Nigri & 75 & $44.4 \%$ \\
Aspergillus section Flavi & 20 & $11.8 \%$ \\
Aspergillus section Fumigati & 13 & $7.7 \%$ \\
Aspergillus section Nidulante & 8 & $4.7 \%$ \\
Aspergillus section Terrei & 5 & $3.0 \%$ \\
Candida species & 48 & $28.4 \%$ \\
Total & 169 & 100 \\
\hline & & \\
\hline Bacterial species & Number of isolates & Percentage (\%) \\
\hline Staphylococcus aereus & 45 & $44.12 \%$ \\
Pseudomonas species & 26 & $25.49 \%$ \\
Klebsiella species & 21 & $20.59 \%$ \\
Eschericha coli & 7 & $6.86 \%$ \\
Proteus specis & 3 & $2.94 \%$ \\
Total & 102 & 100 \\
\hline
\end{tabular}

\section{Discussion}

Otomycosis is troublesome diseases encountered in the otorhinolaryngology department which affect the external auditory canal with infrequent complications involving the middle ear. This infection can be acute or sub acute, and is characterized by itching, earache, blocking sensation and discomfort. Outstanding to the varied microbiological flora of the ear, both fungi and bacteria are involved in otomycosis causing a mixed infection which can be bilateral or unilateral, early diagnosis is of paramount importance to guide proper treatment. In the present study; majority of the patients were in the age group 21 to 40 years and females were affected more than males which is corroborating with other studies showing higher incidence among 21-30 years of age [14, 1821]. This may be because people of this age group are the active age group involved in different activities which expose them to the infectious agent present in the air [22]. We had a female predominance in otitis cases to be $52 \%$ against $48 \%$ in male. Similar findings have been reported by many authors $[5,23,24]$. This may be due to the fact that Female are highly involved in house hold work which exposed them to dusty air containing spores making them vulnerable to the otitis infection [22].

In our study, antibiotic ear drops was the most common predisposing factor (24\%) followed by no cerumen, diabetes, Ear pricking with hard objects, earpiece and swimming. This was in accordance with the study of Fasunla et al. [25], who found $42 \%$ of cases with the same predisposing factor. This study also corroborates with the studies conducted by Rawat Sarita et al. [21] which shows history of using wicks present in $35 \%$ of the cases, followed by association with diabetes in $7 \%$ and swimming history in $4 \%$ cases. Use of hard objects to clean the ear canal leads to trauma in which fungal spores settles and cause infection. Swimming in ponds and rivers is a common way of getting such infection because water trapped in ear canal after bathing in a humid climate can cause fungal infection of the ear [26] and use of antibiotic ear drops act as a risk factor because the antibiotic weakens the normal immunity of the ear and provides a suitable medium for the growth of the fungus [5].

In our study, common presenting symptom was itching in $24 \%$ of cases followed by ear pain $(15.2 \%)$ which is similar to findings by other researchers [20,27]. Inflammation of the ear canal leads to itching and pain and other symptoms like sensation of ear blockage, discharge and decreased hearing may be due to accumulation of fungal debris in the ear canal [27].

Among the 225 cases which showed culture positive, 104 cases showed fungal growth, 53 cases presented bacterial growth and 68 cases showed mixed growth. This our study corroborates with other studies carried by Kulal et al. [20], Rawat et al. [21]. This higher incidence of fungal growth can be attributed to hot and humid climate of the Centre Region of Cameroon.

Most of the fungal ear infections are caused by members of Aspergillus spp like A. niger, A. fumigatus and Candida albicans [20] which is seen in our study having Aspergillus section Nigri as the predominant pathogens in $47.48 \%$ of fungi growth. Species in the section Nigri can grow in cerumen, epithelial scales and debris deep in external auditory canal. This is in accordance with study by Joy et al. [28] and Paulose et al. [7]. Among bacteria, Staphylococcus aureus and Pseudomonas spp. were the predominant isolates. This finding is in accordance with study by Kulal et al. [20]. Bacterial infection of auditory canal may be one of the predisposing factors for development of otomycosis [5]. Mixed bacterial and fungal infections were also reported in a studies carried out in Indian [29, 26, 30].

\section{Conclusion}

The usage of antibiotic ear drops for treatment of otitis external is the major predisposing factor seen in our study with Itching presenting the major symptom in majority of patients. Aspergillus and Candida species are the major fungal isolates and Staphylococcus aureus and Pseudomonas species are major bacterial isolates. Upon clinical suspicion, culture of both bacteria and fungi are required to identify the pathogen, as mixed infections are also seen in cases of otitis externa. 


\section{Conflicts of Interest}

The authors declare no conflict of interest.

\section{Author Contributions}

CBT, JPD conceived and designed the experiments; IAE performed the experiments; JPD and IAE analyzed the data; IAE drafted the manuscript and JPD finalized the paper.

\section{Acknowledgements}

Dzoyem J. P. is thankful to "The World Academy of Sciences (TWAS) for funding this work through the TWAS Research Grant Agreement $\mathrm{N}^{\circ}$ 17-380 RG/BIO/AF/AC_I- FR3240297751. We also thank all the head of departments and medical doctors of the Central and University teaching hospitals for their collaboration during this study.

\section{References}

[1] Carney, A. S. Otitis externa and otomycosis. In: Gleeson $\mathrm{MJ}$ Jones NS, Clarke R, et al. (eds). Scott-Brown's Otolaryngology, Head and Neck Surgery, vol 3, 7th edn. London: Hodder Arnold Publishers; 2008, 3351-7.

[2] Jadhav, V. J., Pal, $\mathrm{M}$ and Mishra, G. S. Etiological significance of Candida albicans in otitis externa. Mycopathologia, 2003, 156: 313-315.

[3] Araiza, J., Canseco, P and Bonifaz, A. Otomycosis: clinical and mycological study of 97 cases. Review of Laryngology Otol Rhinol. 2006, 127: 251-254.

[4] Pradhan, B., Ratna Tuladhar, N and Man Amatya, R. Prevalence of otomycosis in outpatient department of otolaryngology in Tribhuvan University Teaching Hospital, Kathmandu, Nepal. Annals of Otology, Rhinology and Laryngology, 2003, 112: 384-387.

[5] Pontes, Z. B., Silva, A. D, Lima Ede, O., Guerra, M. H., Oliveira, N. M., Carvalho, M. F and Guerra, F. S. Otomycosis: a retrospective study. Brazilian Journal of Otorhinolaryngology, 2009; 75: 367-370.

[6] Kurnatowski, P and Filipiak, A. Otomycosis: prevalence, clinical symptoms, therapeutic procedure. Mycoses, 2001; 44: 472-479.

[7] Paulose, K. O., Khalifa, A. L. S., Shenoy, S. H and Sharma, R. K. Mycotic infection of ear (Otomycosis): Aprospective study. Journal of Laryngology and Otology, 1989; 103: 30-35.

[8] Pakshir, K; Sabayan, B; Javan, H; Karamifar, K. Mycoflora of human external auditory canal in Shiraz, southern Iran. Iran Red Crescent Med J, 2008; 10: 27-29.

[9] Gharaghani, M., Seifi, Z and Mahmoudabadi, A. Z. Otomycosis in Iran: A Review of Mycopathology, 2015; 179: 415-424.

[10] Cheraghsahar, S., Kazemi, S, Birjandi, M., Yarahmadi, M., Mahmoudi, S., Mohammadi, R and Sepahvand, A. Otomycosis in Western Iran: clinical and mycological aspects.
Arch Clin Infect Dis; 2017; 12: e57287.

[11] Viswanatha, B., Sumatha, D and Vijayashree, M. S. Otomycosis in immunocompetent and immunocompromised patients: comparative study and literature review. Ear, Nose and Throat Journal, 2012; 91: 114-121.

[12] Ozcan, K. M., Ozcan M., Karaarslan, A and Karaarslan, F. Otomycosis in Turkey: predisposing factors, aetiology and therapy. Journal of Otolaryngology, 2003; 117: 39-42.

[13] Kujundzic, M, Braut T, Manestar D, Cattunar, A., Malvic, G., Vukelic, J., Puselja, Z and Linsak, D. T. Water related otitis externa. Collegium Antropologicum, 2012; 36: 893-897.

[14] Satish, H. S., Viswanatha, B and Manjuladevi, M. A clinical study of otomycosis: Journal of Dental and Medical Sciences, 2013; 5: 57-62.

[15] Chander, J., Maini, S., Subrahmanyan, S andHanda, A. Otomycosis: A clinico-mycological study and efficacy of mercurochrome in its treatment. Mycopathologia. 1996; 135: $9-12$.

[16] Collee, J. G., Miles, R. S., and Watt, B. Tests for identification of bacteria, in Mackie and McCartney's Practical Medical Microbiology,. Collee J. G, Fraser A. G, Marmion B. P., and Simmons A., Editors, Churchill Livingstone, New York, NY, USA, 1996. pp. 131-145.

[17] Fischer, F and Cook, M. Some opportunistic fungiand yeasts and yeast-like fungi. Fund Diagn Mycol. 1998.

[18] Gokhale, S. J., Suligavi, S. S., Baragundi, D and Manjula, R. Otomycosis: A clinico mycological study. International Journal of Medical Health Sciences, 2013; 2: 218-223.

[19] Haja, A. N., Shaik, K. M. and Siva Subba Rao, P. Mycology of Otomycosis in a tertiary care teaching hospital. Journal of Medical Research and Dental Sciences, 2015; 3: 27-30.

[20] Kulal B., Bhat K. S., Meundi $M$ andKotigadde S. A microbiological study of otomycosis: Indian Journal of Microbiology Research, 2017; 4: 118-125.

[21] Rawat S., Saxena N., Chand E. A., Garg N., Verma V and Sharma K. Cinicomycological study of otomycosis with antifungal drug susceptibility testing of Candida isolates using disk diffusion method in Kota region, Rajasthan, Indian. International Journal of Current Microbiology and Application of Sciences, 2017; 6: 3356-3366.

[22] Kaur, R., Mittal, N., Kakkar, M., Agarwal, A. K and Mathur, M. D. Otomycosis: A clinico-mycological study. Ear, Nose and Throat Journal, 2000; 79: 606-609.

[23] Prasad, S. C., Kotigadde, S., Shekhar, M., Thada, N. D., Prabhu P. D., Souza T andPrasad K. C. Primary Otomycosis in the Indian Subcontinent: Predisposing Factors, Microbiology, and Classification. International Journal of Microbiology, 2012; 04: 1-9.

[24] Adoga, A. S and Idul, A. A. Otomycosis in Jos: Predisposing factors and management. Afr J Med Sci, 2014; 209-213.

[25] Fasunla, J., Ibekwe, T and Onakoya, P. Otomycosis in western Nigeria. Mycoses 2008, 51: 67-70.

[26] Chapparbandi, R. B., Kazi, F. N andAli, K. Otomycosis: An Overview in Hyderabad Karnataka Region. Journal of Evolution of Medical and Dental Sciences. 2014; 3: 1121311216. 
[27] Monalisa, P., BimochProjna P., Banojini P., Sanghamitra, P., Susmita, K. S., Narasimham, M. V and Indrani, M. Clinicomycological Study of Otomycosis with Antifungal Susceptibility Testing Of Fungal Isolates. Journal of Dental and Medical Sciences, 2019; 18: 7-12.

[28] Joy, M. J., Agarwal M. K and Samanth H. C. Mycological and bacteriological studies in otomycosis. Indian Journal of Otolaryngology, 1980; 32: 72-75.
[29] Panchal, P., Pethani., Patel, D., Rathod, S and Shah, P. Analysis of various fungal agents in clinically suspected cases of Otomycosis. Indian Journal of Basic and Applied Medical Research, 2013; 2: 865-869.

[30] Prasanna, V., Hemlata Katiyar, V. M. and Kannan, I. Study of etiological factors, mycological profile and treatment outcome of otomycosis. International Journal of Medical Research Review, 2014; 2: 355-360. 